\title{
Feature-based attention across saccades and immediate postsaccadic selection
}

\author{
Cécile Eymond $^{1} \cdot$ Patrick Cavanagh $^{1,2} \cdot$ Thérèse Collins ${ }^{1}$
}

Published online: 15 April 2016

(C) The Psychonomic Society, Inc. 2016

\begin{abstract}
Before each eye movement, attentional resources are drawn to the saccade goal. This saccaderelated attention is known to be spatial in nature, and in this study we asked whether it also evokes any feature selectivity that is maintained across the saccade. After a saccade toward a colored target, participants performed a postsaccadic feature search on an array displayed at landing. The saccade target either had the same color as the search target in the postsaccadic array (congruent trials) or a different color (incongruent or neutral trials). Our results show that the color of the saccade target did not prime the subsequent feature search. This suggests that "landmark search", the process of searching for the saccade target once the eye lands (Deubel in Visual Cognition, 11, 173-202, 2004), may not involve the attentional mechanisms that underlie feature search. We also analyzed intertrial effects and observed priming of pop-out (Maljkovic \& Nakayama in Memory \& Cognition, 22, $657-672$, 1994) for the postsaccadic feature search: the detection of the color singleton became faster when its color was repeated on successive trials. However, search performance revealed no effect of congruency between the saccade and search targets, either within or across trials, suggesting that the priming of pop-out is specific to target repetitions within the same task and is not seen
\end{abstract}

Cécile Eymond

cecile.eymond@gmail.com

1 Laboratoire Psychologie de la Perception, Université Paris Descartes \& CNRS, Paris, France

2 Department of Psychological and Brain Sciences, Dartmouth College, Hanover, NH, USA for repetitions across tasks. Our results support a dissociation between feature-based attention and the attentional mechanisms associated with eye movement programming.

Keywords Attention $\cdot$ Eye movements $\cdot$ Saccades $\cdot$ Feature-based attention $\cdot$ Priming of pop-out $\cdot$ Visual search

Prior to each eye movement, attentional resources are drawn to the saccade goal (Deubel \& Schneider, 1996; Hoffman \& Subramaniam, 1995; Kowler, Anderson, Dosher, \& Blaser, 1995; Rolfs, Jonikaitis, Deubel, \& Cavanagh, 2011). In this study, we examined whether attention allocated to the saccade target shares any processing with a very different attention system: feature-based attention. Whereas the attention drawn to the saccade target is characterized by spatially localized performance benefits (e.g., Deubel \& Schneider, 1996), feature-based attention is assumed to enhance feature-specific representations throughout the visual field (e.g., Arman, Ciaramitaro, \& Boynton, 2006; Liu \& Mance, 2011). For example, when searching for a red target, red stimuli become salient across the entire visual field (Martínez-Trujillo \& Treue, 2004; Serences \& Boynton, 2007). Here we evaluated whether the attention that is drawn to the saccade target can facilitate a postsaccadic feature search: would the saccade target features prime a visual search presented as the eyes landed? Previous work had reported that feature-based attention and attention drawn to the saccade target did not interact during the interval prior to the saccade: objects that shared features with the saccade target did not receive any attentional benefits (Jonikaitis \& Theeuwes, 2013; White, Rolfs, \& Carrasco, 2013). In the present study, we went further, to assess whether this dissociation is maintained after the execution of the eye movement. If attention drawn to the saccade target primes any feature selectivity, would this feature selectivity spread across 
the visual field once the saccade had landed? The response to this question might help us understand the mechanisms underlying space constancy and the selection processes involved in priming of pop-out.

In our study, participants made a saccade toward a colored target. During saccade execution, this target was removed and replaced by a feature search array. The array had a unique target - for example, a red square among blue squares. We expected the unique-colored element to capture attention and pop out in the array as the most salient (Treisman \& Gelade, 1980). Its selection should be easy, automatic, and parallel. Because the target was defined as the odd item in the array, its color did not have to be specified in advance, and so was set randomly on each trial (Bravo \& Nakayama, 1992). Once the unique target item was detected, the participant had to report a second feature of the target - whether a horizontal bar on the target was at the top or bottom-allowing us to measure how quickly the odd target was located (see Fig. 1). Critically, as is shown in Fig. 1, the pop-out target had either the same color as the saccade target (congruent trial) or a different color (incongruent trial). We examined the effect of congruency on search performance within and across trials. Would the features of the saccade target prime subsequent search targets that shared the same feature?
There is evidence that visual information about salient locations is maintained (Deubel, Schneider, \& Bridgeman, 2002; McConkie \& Currie, 1996; Melcher, 2009) and integrated (Melcher \& Morrone, 2003; Pollatsek, Rayner, \& Collins, 1984; Prime, Tsotsos, Keith, \& Crawford, 2007; Wittenberg, Bremmer, \& Wachtler, 2008) across saccades. But only a few studies have examined the impact of the visual properties of the saccade target on postsaccadic target selection (Hollingworth, Richard, \& Luck, 2008; Richard, Luck, \& Hollingworth, 2008). These studies demonstrated that features of the saccade target do guide corrective saccades. In the Hollingworth et al. study, participants executed a spatially guided saccade toward a colored target embedded within a circular array of differently colored distractors. The whole display was rotated during the saccade so that the eye landed between the target and a distractor. A fast, automatic, and accurate corrective saccade was programmed toward the displaced saccade target. The authors concluded that the postsaccadic selection guiding attention to the new location required the color of the initial target to be maintained across the saccade. Here, we investigated postsaccadic selection from a different viewpoint, and looked at whether the features of the saccade target facilitated a visual search displayed once the eyes had landed.

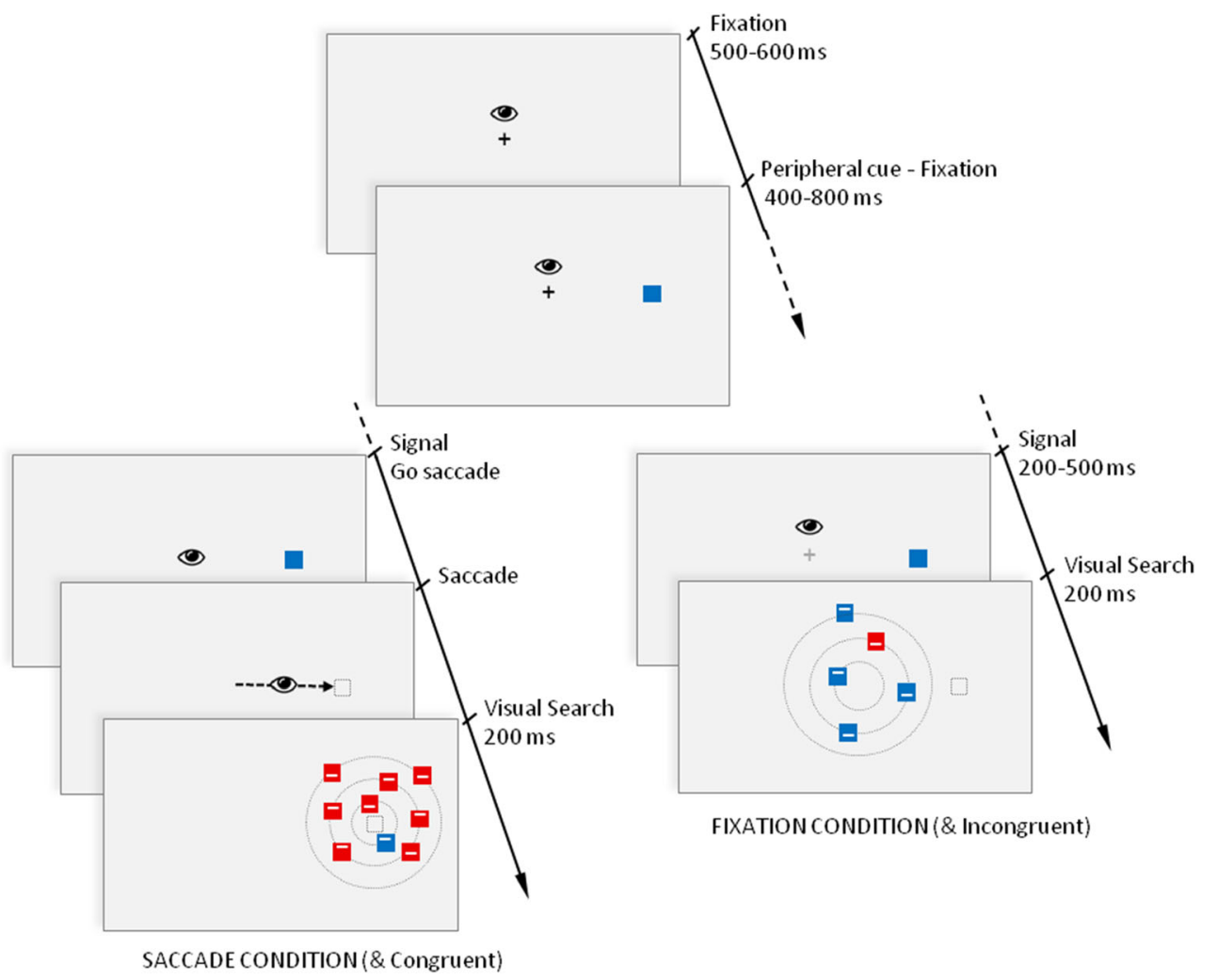

Fig. 1 Experimental design. For both conditions, trials started with a central fixation, followed by presentation of the peripheral stimulus. In the saccade condition (left panels), participants made a saccade toward the peripheral target as soon as the fixation spot disappeared. The search

display was presented after the onset of the saccade and before the eyes landed. In the fixation condition (right panels), participants maintained fixation, and the search display was presented centered at the fovea after a variable delay following the dimming of the fixation dot 


\section{Landmark search and space constancy}

Saccades elicit retinal shifts, but we do not perceive any jumps of the surrounding world. According to the landmark model (Deubel, 2004; Deubel, Bridgeman, \& Schneider, 1998), the realignment of our spatial reference frame is based on the visual information available after each eye movement. The landmark model suggests that a snapshot of the target is taken just prior to the saccade, and once the saccade lands, the visual system searches for that target. Then the target location is used to realign visual coordinates and establish space constancy (Deubel, 2004). We investigated whether feature-based attention plays a role in landmark search. In this framework, attention selects the saccade target that serves as a landmark pattern (Mathôt \& Theeuwes, 2011) and is involved in the search for that target once the eyes land.

The visual properties of the saccade target encoded presaccadically specify what to search for after the saccade (Currie, McConkie, Carlson-Radvansky, \& Irwin, 2000; Deubel, 2004). When the pre- and postsaccadic displays differ, the visual system tends to accept any postsaccadic item close to the presaccadic target location as the landmark for spatial recalibration (Deubel, 2004). If this landmark process is based on a classic visual search, there may be an advantage for any postsaccadic item that matches the saccade target, whatever its location. Thus, our hypothesis is that if the landmark search calls on classic visual search mechanisms, the features of the saccade target should prime processing at all locations sharing the same feature immediately after the saccade. We should therefore observe an advantage for a search target that shares features with the saccade target (congruent trials), relative to search targets that do not (incongruent trials). If attention to the saccade target acts through the same mechanisms as standard visual search, then a color match between the saccade target and the search target should speed the postsaccadic visual search.

\section{Priming of pop-out}

We also examined intertrial priming effects and expected to find priming of pop-out (Maljkovic \& Nakayama, 1994). When the same color singleton is repeated across successive search trials - for example, a red target on several trials in a row-its detection becomes faster than for a nonrepeated target. The processing stages and the mechanisms underlying priming of pop-out are still debated (Becker, 2008b; Kristjánsson \& Campana, 2010; Lamy, Yashar, \& Ruderman, 2010). In our study, we did not assess the postperceptual response-related view that states that priming of pop-out affects decisional processes after a target is selected (Huang, Holcombe, \& Pashler, 2004; Olivers \& Meeter, 2006). In our experiment, the target-defining feature was indeed independent of the response-related feature (Goolsby \& Suzuki, 2001; Maljkovic \& Nakayama, 1994, 2000). According to the attentional view of priming of pop-out, early perceptual and attentional stages are facilitated (Maljkovic \& Nakayama, 1994; Eimer, Kiss, \& Cheung, 2010; Meeter \& Olivers, 2006; Meeter \& Van der Stigchel, 2013). But the nature of the processes that speed attentional shifts to the locations of previously attended targets remains uncertain. Specifically, it is not well known what aspects of attention to the search target on the previous trial enable the priming of pop-out (Ásgeirsson \& Kristjánsson, 2011; Becker, 2008a; Lee, Mozer, \& Vecera, 2009; Maljkovic \& Nakayama, 2000). We investigated whether the nature of the target selection involved in priming of pop-out shares any process in common with the selection of the saccade target, by testing whether attention to the saccade target on the current or the previous trial could modulate the priming of pop-out. We also examined the effect of congruency repetitions over consecutive trials, when the feature relationship between the saccade and search targets is repeated.

In our study, attention drawn to the saccade target may produce priming both within and across trials. If the color of the saccade target were to prime the postsaccadic search for a same-colored target on the current and subsequent trials, it would indicate that the priming, including the priming of pop-out, is based on selection of the target properties, including the case in which the target is the saccade goal. It would also support the hypothesis that landmark search, proposed to explain visual constancy across saccades, calls on the same processes as classic visual search. However, if there were no priming from the saccade target, it would indicate that the priming of pop-out requires prior selection in the same search context, and suggest as well that the landmark search process, if there is such a mechanism, calls on different attentional processes from the feature-based attention underlying visual search.

\section{Method}

\section{Participants}

Seven participants (three men, four women, including the author C.E.) between 25 and 35 years old, with normal or corrected-to-normal vision, took part in the experiment. All participants gave their informed written consent prior to participation. The protocols for the study were approved by the Paris Descartes University Review Board, CERES, in accordance with French regulations and the Declaration of Helsinki. The participants, except for author C.E., were naïve as to the purpose of the study and were compensated $€ 10$ per hour. 


\section{Apparatus and stimuli}

The experiment was programmed in MATLAB, using the Psychophysics and Eyelink Toolbox extensions (Brainard, 1997; Cornelissen, Peters, \& Palmer, 2002). The stimuli were squares measuring $0.62^{\circ}$ per side, displayed in red $(7.59 \mathrm{~cd} /$ $\mathrm{m}^{2}$, CIE $\left.x, y=0.523,0.381\right)$, blue $\left(6.27 \mathrm{~cd} / \mathrm{m}^{2}, x, y=0.194\right.$, $0.205)$, or black $\left(0.11 \mathrm{~cd} / \mathrm{m}^{2}, x, y=0.304,0.364\right)$ on a gray background $(x, y=0.307,0.341)$ with a luminance of $21.3 \mathrm{~cd} /$ $\mathrm{m}^{2}$. The black fixation dot was circular (diameter $0.3^{\circ}$ ). All stimuli were presented at a viewing distance of $53 \mathrm{~cm}$ on a 20 in. CRT monitor screen (spatial resolution of 1,024 $\times$ 768 pixels, vertical refresh rate of $120 \mathrm{~Hz}$ ). Movements of the right eye were recorded using an EyeLink 1000 eyetracker (SR Research Ltd., Mississauga, ON, Canada) operating at $1000 \mathrm{~Hz}$. The participant's head was stabilized by a chinand-forehead rest. Saccades were detected online, and when the latency was more than $2 \mathrm{~s}$, the trial was interrupted and rerun at the end. Manual responses were recorded with a standard keyboard. When no answer was given after $2 \mathrm{~s}$, participants received a message asking them to respond faster, and the trial was also rerun at the end of the session.

\section{Design and procedure}

In the saccade condition, trials began with participants fixating a black dot at one out of four possible locations (at the center of the screen, $1^{\circ}$ from the center in the left direction, or $1^{\circ}$ above or below from the center). After a variable delay of fixation from 500 to $600 \mathrm{~ms}$, a red, blue, or black square target appeared in the right visual field at $12^{\circ}$ eccentricity for $400-800 \mathrm{~ms}$. No specific instruction was given to pay attention to the saccade target color. Participants were instructed to maintain fixation until the signal to make a saccade toward the target, given by the disappearance of the fixation dot. When the saccade onset was detected online (see the Analyses section for onset detection criterion), the target was replaced by a search display for $200 \mathrm{~ms}$ around the saccade target location (see the Results section for the actual presentation duration on refixations). None of the items in the search array were presented at the previous saccade target location. The postsaccadic search display had one square of a unique color (red or blue) among four, eight, or 12 distractors of a different color (blue or red; thus, the display contained five, nine, or 13 total items). The search target was presented equally often at $1^{\circ}$, $2^{\circ}$, or $3^{\circ}$ from the original saccade target location, and the distractor locations were randomly defined on each trial at $1^{\circ}, 2^{\circ}$, or $3^{\circ}$ from the original saccade target location (this means that the search array was displayed within $3^{\circ}$ of the landing position, which is the mean estimated spatial extent of the landmark effect; Deubel, 2004). The task was to find the odd-colored item in the search display and report as quickly as possible the position of a small, white bar at either the top or the bottom of the search target. Participants answered with a keyboard, and response times were recorded. Trials with an incorrect response were discarded and rerun at the end of the block. The search target was always present, and its color was not known in advance, changing randomly across trials.

The fixation condition was identical to the saccade condition, with the following exceptions: participants kept fixation all along. After a variable delay, a peripheral colored square (red, blue, or black) appeared $12^{\circ}$ to the right of fixation for between 400 and $800 \mathrm{~ms}$. The color of the fixation dot changed from black to gray for $200-500 \mathrm{~ms}$, and then the search display (a single red or blue target among blue or red distractors) was presented around the fixation position for $200 \mathrm{~ms}$. No search items coincided with the fixation location (see Fig. 1).

The color relationship between the peripheral target and the search array target was either congruent (both same color, either red or blue), incongruent (different colors, with the peripheral target matching the distractors), or neutral (black peripheral target and a red or blue search target). There were four target types (target red or blue, with a small white bar at the top or the bottom) for each of the four possible fixation positions and each search target eccentricity $\left(1^{\circ}, 2^{\circ}\right.$, or $\left.3^{\circ}\right)$. These 48 trial types were tested for each combination of the three congruency levels (congruent, incongruent, or neutral) and the three display sizes (four, eight, or 12 distractors), for a total of 432 trials per session, distributed randomly in six blocks of 72 trials with short breaks between the blocks. Each participant completed two sessions in the fixation condition and two sessions in the saccade condition. Each session lasted approximately $1 \mathrm{~h}$ (trials aborted were rerun at the end of each block). Sessions were run separately, and their order was counterbalanced across participants.

\section{Analyses}

The response saccade was identified online by means of a spatial boundary criterion: it was the first saccade that left a circular region with a radius of $2^{\circ}$ around fixation. The response saccade onset and offset (landing) were recorded online using the EyeLink default velocity criterion of $30 \%$ s. Eye position was recorded from the time of fixation onset when the trial began to the time of the manual response.

In an offline screen procedure, we checked that the response saccade triggered the presentation of the search display, and that the search display was on before the eye landed. We further analyzed offline the response saccade properties, to check several criteria for correct trials. Specifically, saccade trials were excluded from the analysis according to the following criteria: (a) anticipatory saccades (latency $<100 \mathrm{~ms}$ ), (b) delayed saccades (latency $>800 \mathrm{~ms}$ ), (c) gaze having deviated by more than $2^{\circ}$ from the fixation dot at the time of the saccade onset, or (d) a landing position more than $3^{\circ}$ from the saccade 
target. Fixation trials were discarded when the gaze fell outside the $2^{\circ}$ radius around fixation at the time of the display onset.

In the saccade condition, the saccade latency was defined as the interval between the offset of the fixation dot (the signal to make a saccade) and the initiation of the response saccade. Reaction times were given by the time difference between the saccade landing and the keypress time. In the fixation condition, reaction times were measured as the delay between the onset of the display and the keypress time.

Repeated measures analyses of variance (ANOVAs) were performed for statistical comparisons, with a significance criterion set at $p<.05$.

\section{Results}

\section{Within trials}

Reaction times were analyzed for correct trials only. For the saccade condition, $5.2 \%$ of the trials were excluded offline on the basis of the screening criteria (see the Method section), and the error rate for the remaining trials was $5.4 \%$. For the fixation condition, $1.2 \%$ of the trials were excluded for gaze deviations, and the error rate was $4.0 \%$ on the remaining correct trials. The mean saccade latency in the saccade condition, for the correct trials, was $216 \mathrm{~ms}( \pm 10 \mathrm{~ms}, S E M)$. The postsaccadic array was displayed for $200 \mathrm{~ms}$ after online saccade detection. Offline analyses revealed that the array was presented for a mean of $183 \mathrm{~ms}( \pm 2 \mathrm{~ms}, S E M)$ after saccade landing.

Reaction times were measured as a function of the display size (Fig. 2) and are plotted according to congruency in Fig. 3. In the saccade condition, participants were significantly faster to identify the search target, with a $27-\mathrm{ms}$ mean difference (range: 14-48 ms) relative to the fixation condition (Fig. 2) $[F(1,6)=31.304, p=.001]$. A possible explanation for the difference is that in the saccade condition, attention was captured at the search target location right upon saccade landing. In contrast, in the fixation condition, attention was first shifted

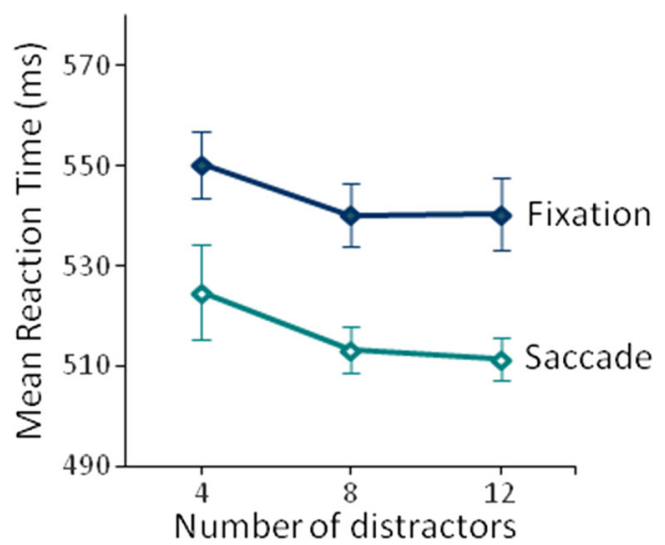

Fig. 2 Mean reaction times as a function of display size, in the fixation and saccade conditions. Error bars indicate \pm 1 within-subjects error

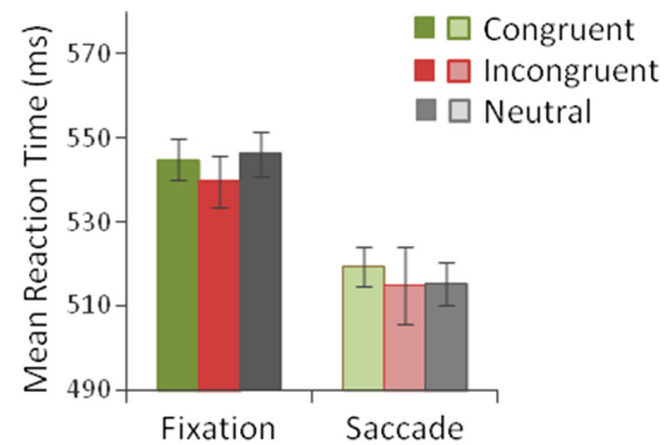

Fig. 3 Mean reaction times for congruent, incongruent, and neutral trials, in the fixation and saccade conditions. Error bars indicate \pm 1 withinsubjects error

to the peripheral square and then back to the search array presented around the fixation point. The attentional disengagement from the peripheral square may have slowed the responses in the fixation condition.

The number of distractors had a significant impact on search performance, similarly in both the fixation and saccade conditions (Fig. 2). Reaction times decreased as the number of distractors increased $[F(2,12)=7.487, p<.01]$, replicating the classic results for a color oddball search in which the target and distractors colors change unpredictably from trial to trial (Bravo \& Nakayama, 1992). As was proposed by these authors, the target probably becomes more salient when there are more distractors.

We also found the classic effect of search target eccentricity $[F(2,12)=166.4, p<.001]$ (Carrasco \& Katz, 1992), with reaction times increasing with eccentricity similarly in both the fixation and saccade conditions (with larger distance, respectively, between the fixation point and the search target or between the saccade and search targets) $[F(2,12)=1.445, p>.1]$.

Overall, congruency had no effect on reaction times (Fig. 3) $[F(2,12)=2.219, p>.1]$ and did not interact with condition [fixation vs. saccade: $F(2,12)=0.973, p>.1$ ], display size $[F(4,24)=1.343, p>.1]$, or both $[F(4,24)=$ $1.002, p>.1]$, or with eccentricity $[F(4,24)=0.065, p>.5]$. A color match between the peripheral target and the search target did not lead to any benefit in the subsequent visual search. A retrospective analysis indicated that the small observed effect of congruency (congruent trials were numerically $5 \mathrm{~ms}$ slower than incongruent ones) would need 324 participants to reach significance. Even if we were to confirm this effect on a very large population, it would still be very small and opposite to all reported effects of congruency. Our conclusion is that the color of the saccade target has little or no effect on the postsaccadic search task.

\section{Across trials}

Priming of pop-out We compared performance on consecutive trials and observed the classic priming effect of the search 
target (Maljkovic \& Nakayama, 1994). Specifically, we observed a decrease in reaction times when the search target had the same color on two successive trials $[F(1,6)=47.402, p<$ $.001]$. This effect did not differ between saccade and fixation trials $[F(1,6)=0.122, p>.5]$. The mean reductions were $28 \mathrm{~ms}$ for four distractors, $9 \mathrm{~ms}$ for eight distractors, and $7 \mathrm{~ms}$ for 12 distractors. Maljkovic and Nakayama reported a 60-ms advantage for a search array with three items (two distractors) that were more widely spaced - at $4^{\circ}$ to $5^{\circ}$ from fixation, as opposed to $1^{\circ}$ to $3^{\circ}$ in our display. These factors could explain the smaller effect we found here. Indeed, display size interacted significantly with priming of pop-out $[F(2$, $12)=9.843, p<.005]$ : the priming effect decreased as the number of distractors increased (see Fig. 4). This replicates classic results for priming in color oddball search (Meeter \& Olivers, 2006).

When the color of the search target remained the same across increasing numbers of trials, the reaction time advantage (priming of pop-out averaged across set sizes) also increased (shown in Fig. 5 for up to four repetitions [e.g., red-red-red-red], as compared to trials in which the last target color differed from the previous ones [e.g., blueblue-blue-red]).

Effect of congruency A color match between the saccade and search targets (congruent trials) had no impact on the priming of pop-out relative to incongruent trials, averaged across all display sizes $[F(2,12)=0.415, p>.5]$. The sizes of the priming of pop-out effects for congruent versus incongruent trials were $21 \pm 3$ versus $15 \pm 6 \mathrm{~ms}$, respectively, in the fixation condition, and $13 \pm 3$ versus $15 \pm 3 \mathrm{~ms}$, in the saccade condition (errors indicate $\pm 1 S E M$ ). Also, the congruency between the saccade and search targets of the previous trial had no impact on priming of pop-out $[F(2,12)=0.429, p>.5]$. Overall, selection of the saccade target on the current or on the previous trial did not modulate the strength of the priming of pop-out. The current search target was primed only by previous search targets.

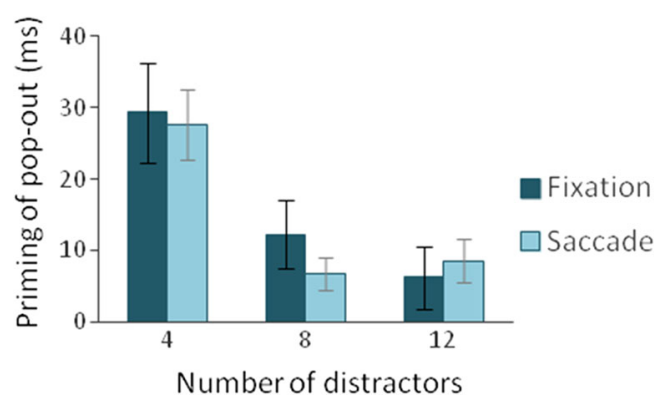

Fig. 4 Priming of pop-out according to the number of distractors present in the display. The priming of pop-out is the mean reaction time difference between trials with repeated and trials with nonrepeated target colors. Error bars indicate $\pm 1 S E M$

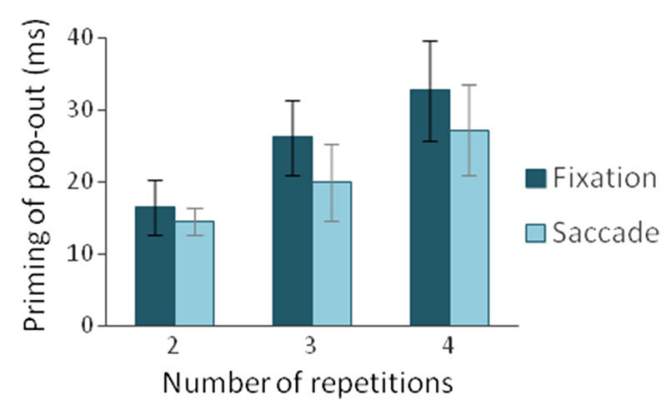

Fig. 5 Priming of pop-out as a function of the number of repetitions. The priming of pop-out is the mean reaction time difference between $n$ repetitions of the same-colored target (e.g., red-red-red-red) and $n-1$ repetitions followed by a trial with a reversed color (e.g., blue-blue-blue-red). Error bars indicate $\pm 1 S E M$

Congruency repetition Priming of pop-out was also not influenced by the repetition of congruency, averaged across all display sizes $[F(1,6)=0.025, p>.5]$. We observed no interaction with the condition [fixation vs. saccade: $F(1,6)=$ $4.539, p>.05$ ] or with the level of congruency — congruent, incongruent, or neutral $[F(2,12)=2.372, p>.1]$. Particularly, the sizes of the priming of pop-out intertrial effects were similar when the current and previous trials were both congruent and when the current trial was congruent and the previous one was not (either incongruent or neutral). Congruency had no effect on priming of pop-out even when the feature relationship itself was primed.

\section{Discussion}

In this study, we investigated whether the landmark process, suggested as a search for the saccade target by the visual system once the eye lands (Deubel, 2004), is related to the classic mechanisms underlying visual search. To do so, participants performed a postsaccadic visual search, and we evaluated the effect of the feature relationship between the saccade target and the search target — congruency — on search performance. The unique-colored element popped out (Treisman \& Gelade, 1980) in the array displayed around either the landing position (saccade condition) or the fixation position (fixation condition). Our results replicated the classic decrease in reaction times as the number of distractors increased, which is typical for an oddball search when the unique-colored target changes unpredictably across trials (Bravo \& Nakayama, 1992). Congruency between the saccade target color and the oddball target color did not influence search performance in either the saccade or the fixation condition. Participants were just as fast on congruent trials (color match between the saccade target and the search target) as on incongruent trials (color match between the saccade target and the distractors) and neutral trials (saccade target color did not match the color of either the search target or the distractors). These results 
suggest that the saccade target features did not prime the subsequent feature search.

We also examined intertrial priming effects to see whether the classic priming of pop-out (Maljkovic \& Nakayama, 1994) was present across successive search trials and whether the attention to the saccade target on the current trial and on the previous trial modulated this priming. Our results did replicate the intertrial priming of pop-out (Maljkovic \& Nakayama, 1994), with the classic decrease in magnitude as the number of distractors increased (Meeter \& Olivers, 2006). The reaction time advantage also increased with the number of target repetitions (Maljkovic \& Nakayama, 1994). However, the size of the priming of pop-out effect was not modulated by the color of the saccade target in either the current or the previous trial. Furthermore, repeated congruency did not influence priming of pop-out, suggesting that the saccade target features did not interact with the priming of pop-out.

To sum up, we found that the color of the saccade target had no impact on search performance; the saccade target did not prime the search target either within or across trials. These results suggest that the selective process involved in the postsaccadic feature search task is independent of the attention drawn to the saccade target.

In previous studies (Hollingworth et al., 2008; Richard et al., 2008) that have found an effect of congruency between the saccade target and postsaccadic feature-based selection, participants made a saccade toward a colored target that was already situated within an array. The authors examined the fast corrective saccade that occurred when the target was missed due to the displacement of the array during the execution of the eye movement, and the second saccade was preferentially directed to a congruent stimulus. The selection of the congruent stimulus for corrective-saccade planning may have relied on memory resources not involved in our experiment. Indeed, Hollingworth et al. proposed that the target color had to be maintained in visual working memory to discriminate and find the target after the eye movement. In our design, the target feature was also not known in advance, but only a single saccade target was presented before, and the multiple array was displayed at the saccade landing.

Jonikaitis and Theeuwes (2013) and White et al. (2013) examined the effect of congruency between a saccade target and a visual probe presented prior to the saccade. They reported no congruency effect between attention to the saccade target and presaccadic feature-based attention: no featurespecific selection was evoked in the visual field. Nonetheless, during saccade preparation, White and colleagues did observe an adaptive deployment of feature-based attention that was contingent on recent experience, when attentional resources were distributed to unattended locations with potentially relevant features. They found that a presaccadic probe that shared the saccade target feature revealed higher sensitivity only when the saccade target and the probe had been congruent in the previous trial, regardless of the repetition of the feature. But in the $50 \mathrm{~ms}$ preceding saccade onset, this effect was no longer present. We have extended these earlier presaccadic findings to report no congruency effect after the execution of an eye movement-no spatial spread of attention biased by the saccade target properties. We found no evidence of any interaction between postsaccadic selective attention (pop-out visual search) and the attention drawn to the saccade target.

\section{Priming of pop-out}

When the same unique target feature is repeated in successive trials, its detection is facilitated relative to nonrepetition trials, a phenomenon known as priming of pop-out (Maljkovic \& Nakayama, 1994). The efficiency of selecting and reporting the single salient target is modulated by the properties of the preceding search arrays, but the nature of this modulation is still a matter of debate (Becker, 2008b; Kristjánsson \& Campana, 2010; Lamy et al., 2010). The main theoretical account stipulates that early perceptual processing of the feature is facilitated, resulting in faster attentional selection of the repeated target (Eimer et al., 2010; Maljkovic \& Nakayama, 1994; Meeter \& Olivers, 2006; Meeter \& Van der Stigchel, 2013). It remains unclear what aspects of attention to the search target on the previous trial actually speed the attentional selection on the current trial (Ásgeirsson \& Kristjánsson, 2011; Becker, 2008a; Lee et al., 2009; Maljkovic \& Nakayama, 2000).

Priming of pop-out was observed in our data, with a reaction time advantage for repeated search target colors as compared to nonrepeated colors. The magnitudes of the priming of pop-out were similar in the saccade and fixation conditions, and congruency between the saccade and search target features had no impact on priming of pop-out. Selection of the saccade target on the current and previous trials did not modulate the priming of pop-out. This result suggests that the priming of pop-out depends on the selection process involved in the pop-out task itself, not on any general selection of target properties across concurrent tasks, in line with previous work (Goolsby \& Suzuki, 2001; Kristjánsson, Saevarsson, \& Driver, 2013). The attentional mechanisms involved in the selection of an isolated saccade target appear to be different from feature-based attention. Our results support a dissociation between the selective processes involved in priming of pop-out and the attentional processes associated with programming eye movements.

\section{Landmark search and space constancy}

To explain our stable perception of the world across eye movements, the landmark model proposes that the visual system assumes that the world remains unchanged during an eye movement. Following a saccade, the visual system would 
require only rediscovery of a selected spatial landmark - typically the saccade target - to recalibrate the spatial coordinate frame (Deubel, 2004). Each saccade toward a target would therefore be followed, once the saccade lands, by a search for that target, and there would be no need for specific predictions (efference copy) about the consequences of our eye movements. In our study we investigated whether the landmark search process was related to a classic feature-based search (Treisman \& Gelade, 1980). We found that the saccade target did not prime the postsaccadic search target, and since we did find priming of pop-out across trials, reaction times were probably not reduced to the floor. We therefore conclude that landmark search does not call on processes in common with a standard visual search.

Our results suggest that the classic mechanisms underlying feature search may not operate at saccade landing to discover the saccade target, and that the existence of postsaccadic search for a target to ensure space constancy could be challenged. But the question of whether a landmark search might take part in visual stability still remains. Evidence for the postsaccadic search for landmarks arises from the "landmark effect" (Deubel et al., 1998; Deubel, Schneider, \& Bridgeman, 1996), which shows that distractors can be perceived postsaccadically as the target when they best match a saccade target that is no longer present when the eyes land. Landmark search is an alternative explanation to the remapping explanation for space constancy, in which spatial updating of a few attended targets is based on the motor signal of an upcoming saccade (Cavanagh, Hunt, Afraz, \& Rolfs, 2010; Duhamel, Colby, \& Goldberg, 1992; Rolfs et al., 2011; Wurtz, 2008). According to remapping, the visual system predicts the postsaccadic location of the target from the oculomotor efference copy, and if the target is not in a tolerance region around the predicted location after the eyes land, it is perceived as having moved (Collins, Rolfs, Deubel, \& Cavanagh, 2009; Wexler \& Collins, 2014). Both the remapping and landmark explanations might coexist, because dissociated mechanisms have been proposed to explain stability for action and for perception (Bays \& Husain, 2007).

Alternative explanations might be considered for the lack of influence of the saccade target properties on postsaccadic visual search. For example, after a saccade, the visual system may prioritize the reestablishment of space constancy before performing any other attentional tasks. In other words, the postsaccadic landmark search could have been completed before the feature search started. It is also possible that a substantial change between the pre- and postsaccadic visual scenes might affect the landmark search, to the extent that it would be abandoned as irrelevant. Furthermore, in our experiment no element was present at the original saccade target position in the search array. A recent study revealed that spatial updating of attention across eye movements depends critically on the continued presence of an object at the attended location both before the saccade and after its landing (Lisi, Cavanagh, \& Zorzi, 2015). In our study, without this spatial continuity, attentional benefits might not have been transferred from the saccade target to the subsequent search elements.

In summary, in the present study we investigated the interplay between the attention allocated to the saccade target and a postsaccadic feature search. We found no evidence that the color of the saccade target facilitated a subsequent visual search for a target of the same color, either within or across trials. Classic priming of pop-out was observed between the search features of the sequential pop-out trials, but the reaction time advantage of the priming of pop-out was not affected by congruency to the saccade target color on the current or previous trials. We conclude that any landmark search proposed to explain space constancy - if it occurs - does not involve the attentional mechanisms that underlie feature search. Our results also suggest that the priming of pop-out depends on the pop-out search context itself, and is not supported by general feature-based attention to the target properties in other concurrent tasks. Our results support a dissociation between featurebased attention and the attentional processes associated with programming eye movements.

Author note The research leading to these results received funding from the European Research Council under the European Union's Seventh Framework Program (FP7/2007-2013)/ERC Grant Agreement No. AG324070 to P.C. We thank Lionel Granjon for his help with analysis of the eye movement data. The authors have no commercial relationships to report.

\section{References}

Arman, A. C., Ciaramitaro, V. M., \& Boynton, G. M. (2006). Effects of feature-based attention on the motion aftereffect at remote locations. Vision Research, 46, 2968-2976.

Ásgeirsson, Á. G., \& Kristjánsson, Á. (2011). Episodic retrieval and feature facilitation in intertrial priming of visual search. Attention, Perception, \& Psychophysics, 73, 1350-1360. doi:10.3758/s13414011-0119-5

Bays, P. M., \& Husain, M. (2007). Spatial remapping of the visual world across saccades. NeuroReport, 18, 1207-1213.

Becker, S. I. (2008a). The mechanism of priming: Episodic retrieval or priming of pop-out? Acta Psychologica, 127, 324-339. doi:10. 1016/j.actpsy.2007.07.005

Becker, S. I. (2008b). The stage of priming: Are intertrial repetition effects attentional or decisional? Vision Research, 48, 664-684. doi: 10.1016/j.visres.2007.10.025

Brainard, D. H. (1997). The Psychophysics Toolbox. Spatial Vision, 10, 433-436. doi:10.1163/156856897X00357

Bravo, M. J., \& Nakayama, K. (1992). The role of attention in different visual-search tasks. Perception \& Psychophysics, 51, 465-472. doi: 10.3758/BF03211642

Carrasco, M., \& Katz, S. M. (1992, April). The effect of target position in a feature visual search task. Paper presented at the 63rd Annual Meeting of the Eastern Psychological Association, Boston, MA.

Cavanagh, P., Hunt, A. R., Afraz, A., \& Rolfs, M. (2010). Visual stability based on remapping of attention pointers. Trends in Cognitive Sciences, 14, 147-153. doi:10.1016/j.tics.2010.01.007 
Collins, T., Rolfs, M., Deubel, H., \& Cavanagh, P. (2009). Post-saccadic location judgments reveal remapping of saccade targets to nonfoveal locations. Journal of Vision, 9(5), 29:1-9. doi:10.1167/9.5.29

Cornelissen, F. W., Peters, E. M., \& Palmer, J. (2002). The Eyelink Toolbox: Eye tracking with MATLAB and the Psychophysics Toolbox. Behavior Research Methods, Instruments, \& Computers, 34, 613-617. doi:10.3758/BF03195489

Currie, C. B., McConkie, G. W., Carlson-Radvansky, L. A., \& Irwin, D. E. (2000). The role of the saccade target object in the perception of a visually stable world. Perception \& Psychophysics, 62, 673-683.

Deubel, H. (2004). Localization of targets across saccades: Role of landmark objects. Visual Cognition, 11, 173-202.

Deubel, H., Bridgeman, B., \& Schneider, W. X. (1998). Immediate postsaccadic information mediates space constancy. Vision Research, 38, 3147-3159.

Deubel, H., \& Schneider, W. X. (1996). Saccade target selection and object recognition: Evidence for a common attentional mechanism. Vision Research, 36, 1827-1837. doi:10.1016/0042-6989(95) 00294-4

Deubel, H., Schneider, W. X., \& Bridgeman, B. (1996). Postsaccadic target blanking prevents saccadic suppression of image displacement. Vision Research, 36, 985-996.

Deubel, H., Schneider, W. X., \& Bridgeman, B. (2002). Transsaccadic memory of position and form. Progress in Brain Research, 140, 165-180. doi:10.1016/S0079-6123(02)40049-0

Duhamel, J. R., Colby, C. L., \& Goldberg, M. E. (1992). The updating of the representation of visual space in parietal cortex by intended eye movements. Science, 255, 90-92.

Eimer, M., Kiss, M., \& Cheung, T. (2010). Priming of pop-out modulates attentional target selection in visual search: Behavioral and electrophysiological evidence. Vision Research, 50, 1353-1361.

Goolsby, B. A., \& Suzuki, S. (2001). Understanding priming of colorsingleton search: Roles of attention at encoding and retrieval. Perception \& Psychophysics, 63, 929-944. doi:10.3758/ BF03194513

Hoffman, J. E., \& Subramaniam, B. (1995). The role of visual attention in saccadic eye movements. Perception \& Psychophysics, 57, 787795. doi:10.3758/BF03206794

Hollingworth, A., Richard, A. M., \& Luck, S. J. (2008). Understanding the function of visual short-term memory: Transsaccadic memory, object correspondence, and gaze correction. Journal of Experimental Psychology: General, 137, 163-181. doi:10.1037/ 0096-3445.137.1.163

Huang, L., Holcombe, A. O., \& Pashler, H. (2004). Repetition priming in visual search: Episodic retrieval, not feature priming. Memory \& Cognition, 32, 12-20. doi:10.3758/BF03195816

Jonikaitis, D., \& Theeuwes, J. (2013). Dissociating oculomotor contributions to spatial and feature-based selection. Journal of Neurophysiology, 110, 1525-1534.

Kowler, E., Anderson, E., Dosher, B. A., \& Blaser, E. (1995). The role of attention in the programming of saccades. Vision Research, 35, 1897-1916. doi:10.1016/0042-6989(94)00279-U

Kristjánsson, Á., \& Campana, G. (2010). Where perception meets memory: A review of repetition priming in visual search tasks. Attention, Perception, \& Psychophysics, 72, 5-18. doi:10.3758/APP.72.1.5

Kristjánsson, Á., Saevarsson, S., \& Driver, J. (2013). The boundary conditions of priming of visual search: From passive viewing through task-relevant working memory load. Psychonomic Bulletin \& Review, 20, 514-521. doi:10.3758/s13423-013-0375-6

Lamy, D., Yashar, A., \& Ruderman, L. (2010). A dual-stage account of inter-trial priming effects. Vision Research, 50, 1396-1401. doi:10. 1016/j.visres.2010.01.008

Lee, H., Mozer, M. C., \& Vecera, S. P. (2009). Mechanisms of priming of pop-out: Stored representations or feature-gain modulations? Attention, Perception, \& Psychophysics, 71, 1059-1071. doi:10. 3758/APP.71.5.1059
Lisi, M., Cavanagh, P., \& Zorzi, M. (2015). Spatial constancy of attention across eye movements is mediated by the presence of visual objects. Attention, Perception, \& Psychophysics, 77, 1159-1169.

Liu, T., \& Mance, I. (2011). Constant spread of feature-based attention across the visual field. Vision Research, 51, 26-33.

Maljkovic, V., \& Nakayama, K. (1994). Priming of pop-out: I. Role of features. Memory \& Cognition, 22, 657-672. doi:10.3758/ BF03209251

Maljkovic, V., \& Nakayama, K. (2000). Priming of popout: III. A shortterm implicit memory system beneficial for rapid target selection. Visual Cognition, 7, 571-595. doi:10.1080/135062800407202

Martínez-Trujillo, J. C., \& Treue, S. (2004). Feature-based attention increases the selectivity of population responses in primate visual cortex. Current Biology, 14, 744-751. doi:10.1016/j.cub.2004.04.028

Mathôt, S., \& Theeuwes, J. (2011). Visual attention and stability. Philosophical Transactions of the Royal Society B, 366, 516-527.

McConkie, G. W., \& Currie, C. B. (1996). Visual stability across saccades while viewing complex pictures. Journal of Experimental Psychology: Human Perception and Performance, 22, 563-581. doi:10.1037/0096-1523.22.3.563

Meeter, M., \& Olivers, C. N. L. (2006). Intertrial priming stemming from ambiguity: A new account of priming in visual search. Visual Cognition, 13, 202-222.

Meeter, M., \& Van der Stigchel, S. (2013). Visual priming through a boost of the target signal: Evidence from saccadic landing positions. Attention, Perception, \& Psychophysics, 75, 1336-1341.

Melcher, D. (2009). Selective attention and the active remapping of object features in trans-saccadic perception. Vision Research, 49, 1249 1255.

Melcher, D., \& Morrone, M. C. (2003). Spatiotopic temporal integration of visual motion across saccadic eye movements. Nature Neuroscience, 6, 877-881.

Olivers, C. N. L., \& Meeter, M. (2006). On the dissociation between compound and present/absent tasks in visual search: Intertrial priming is ambiguity driven. Visual Cognition, 13, 1-28. doi:10.1080/ 13506280500308101

Pollatsek, A., Rayner, K., \& Collins, W. E. (1984). Integrating pictorial information across eye movements. Journal of Experimental Psychology: General, 113, 426-442.

Prime, S., Tsotsos, L., Keith, G., \& Crawford, J. (2007). Visual memory capacity in transsaccadic integration. Experimental Brain Research, 180, 609-628.

Richard, A. M., Luck, S. J., \& Hollingworth, A. (2008). Establishing object correspondence across eye movements: Flexible use of spatiotemporal and surface feature information. Cognition, 109, 66-88. doi:10.1016/j.cognition.2008.07.004

Rolfs, M., Jonikaitis, D., Deubel, H., \& Cavanagh, P. (2011). Predictive remapping of attention across eye movements. Nature Neuroscience, 14, 252-256.

Serences, J. T., \& Boynton, G. M. (2007). Feature-based attentional modulations in the absence of direct visual stimulation. Neuron, 55, 301-312.

Treisman, A. M., \& Gelade, G. (1980). A feature-integration theory of attention. Cognitive Psychology, 12, 97-136. doi:10.1016/00100285(80)90005-5

Wexler, M., \& Collins, T. (2014). Orthogonal steps relieve saccadic suppression. Journal of Vision, 14(2), 13:1-9. doi:10.1167/14.2.13

White, A., Rolfs, M., \& Carrasco, M. (2013). Adaptive deployment of spatial and feature-based attention before saccades. Vision Research, $85,26-35$.

Wittenberg, M., Bremmer, F., \& Wachtler, T. (2008). Perceptual evidence for saccadic updating of color stimuli. Journal of Vision, 8(14), 9:19. doi:10.1167/8.14.9

Wurtz, R. H. (2008). Neural mechanisms of visual stability. Vision Research, 48, 2070-2089. 